\title{
Puromycin Hydrochloride
}

National Cancer Institute

\section{Source}

National Cancer Institute. Puromycin Hydrochloride. NCI Thesaurus. Code C75148.

The hydrochloride salt form of puromycin, an aminoglycoside antibiotic isolated from the bacterium Streptomyces alboniger. Acting as an analog of the 3 ' terminal end of aminoacyl-tRNA, puromycin incorporates itself into a growing polypeptide chain and causes its premature termination, thereby inhibiting protein synthesis. This agent has antimicrobial, antitrypanosomal, and antineoplastic properties; it is used as an antibiotic in cell culture. 